\title{
Harvesting-Transmission-Harvesting Mode for Cognitive Radio Networks with Energy Harvesting Maximization
}

\author{
He Xiao, Hong Jiang, ${ }^{*}$ and Li-Ping Deng \\ School of Information Engineering, Southwest University of Science and Technology, Mianyang 621010, China
}

(Received September 3, 2021; accepted October 18, 2021)

Keywords: energy harvesting, underlay, throughput maximization, convex optimization, throughput constraint

This paper presents a new cognitive radio network (CRN) framework with radio frequency (RF) energy harvesting, namely, RF-powered cognitive radio networks (RF-CRNs), wherein multiple secondary users (SUs) harvest energy from the RF signals of primary users (PUs) all the time except when transmitting data in the allocated time, namely, the "harvestingtransmission-harvesting" mode. In this way, the energy harvested by each SU can be maximized until the next data transmission. Also, the total energy consumed by the SUs must be less than or equal to the total harvested energy (the energy causality constraint), while the transmit power of SUs must be restricted to protect the PUs from interference (collision constraint). Finally, under the satisfaction of quality of service of SUs (throughput constraint), our goal is to determine the optimal transmitting time and power allocation that maximize the achievable throughput in the RF-CRNs. We achieved the optimal result by transforming the optimization problem into a convex optimization problem and then applying Lagrange multiplier methods. Extensive performance evaluations showed the efficiency of the proposed algorithm.

\section{Introduction}

Nowadays, the problems of spectrum efficiency (SE) and energy efficiency (EE) in wireless networks must be solved urgently, especially in the $5 \mathrm{G}$ era. Cognitive radio networks (CRNs) with energy harvesting (EH) (EH-CRNs) have emerged as a promising way to address the problems of SE and EE while responding to the call for green communication at the same time. ${ }^{(1-6)}$ Through opportunistic spectrum access, a secondary CRN can opportunistically access the spectrum licensed to a primary network.

The performance of wireless networks is greatly limited by the finite capacity of the battery. Powering wireless nodes with energy scavenged from ambient sources such as heat, light, waves, and wind enables wireless nodes to run permanently without the cost of external power cables and recharging or the need to replace the battery periodically. Radio frequency (RF) EH allows a wireless node to harvest and convert electromagnetic waves from ambient RF sources (e.g., TV and radio towers, and cellular base stations) into energy, which can provide energy for many emergency scenes or remote locations that cables cannot reach. ${ }^{(5)}$ 
Compared with ambient EH, RF EH is more flexible and sustainable as the RF signals radiated by ambient transmitters are consistently available. ${ }^{(5)} \mathrm{RF}$ energy sources can mainly be categorized into intended RF and non-intended RF. Intended RF can power nodes that require a predictable and large amount of energy due to on-demand supply and directional transmission. Non-intended RF signals include most of the radiation from nearby RF sources such as cellular base stations, primary users (PUs), and other secondary sources. Such radiation is freely available and not dedicated, and is mainly used to power low-power networks such as sensor networks. By employing EH technology, RF signals radiated by PUs no longer need be regarded as interference for secondary users (SUs) but as a continuous energy source for EH.

\subsection{Related work and motivation}

In the present study, SUs in RF-CRNs mainly operate in three different modes: interweave, overlay, and underlay. ${ }^{7-21)}$ In interweave mode, SUs first harvest energy and then opportunistically access the licensed spectrum for data transmission when PUs are detected as inactive. The SUs work in harvesting-sensing-transmitting mode, where much time and energy are spent on spectrum sensing. Once the PUs retake the channel, the SUs have to interrupt the transmission and give up the channel. ${ }^{(7-12)}$

In overlay mode, given that the SUs have knowledge of the PUs' transmit sequence and encoding scheme, the SUs harvest energy to serve both PUs and SUs. This requires excellent cooperation between PUs and SUs, which is difficult to achieve in practice. ${ }^{(18-21)}$

In contrast to the high energy consumption and discontinuous data transmission in interweave mode and the necessary collaboration of PU and SU in overlay mode, in underlay mode, an SU can transmit along with a PU as long as the secondary interference at the primary receiver remains below an acceptable threshold. Zheng's group presented a novel structure for underlay RF-CRNs. ${ }^{(13)}$ This structure had only one SU transmitter-receiver pair in the RF-CRNs, where the SU receiver had a fixed power supply, while the SU transmitter was powered by an energy harvester that harvested green energy from the signals of the PU transmitter. The same group extended the RF-CRNs to incorporate SUs, wherein all SUs first harvested energy for a fixed duration and then transmitted data using the harvested energy to one access point for the remainder of the period using the code-division multiple access (CDMA) method. ${ }^{(14)}$ Recently, a wireless-powered underlay CRN has been developed, where SUs first harvested energy in the downlink wireless power transfer phase and then used the energy for data transmission in the uplink wireless information transmission phase, adopting time-division multiple access (TDMA). To expand network coverage and reduce the transmission power of SUs in underlay mode, a new wireless EH protocol designed for an underlay cognitive relay network with multiple PU transceivers was proposed in Ref. 16. A green coexistence paradigm for underlay multi-hop EH-CRNs has been formulated in which all the secondary nodes capture energy from the PUs. ${ }^{(17)}$ The authors in Ref. 17 solved the end-to-end throughput maximization problem subject to the energy causality and interference power constraints. With the recent increase in the number of hops, the time delay and outage probability will increase accordingly. ${ }^{(21-31)}$ 
In underlay RF-CRNs, SUs harvest energy and transmit data without interruption by coexisting with the PUs under an interference constraint. SUs take PUs as a stable energy source for sustainable $\mathrm{EH}$ through working in the harvesting-transmitting-inactive mode, in which SUs are always inactive and do nothing after transmitting data at the allocated time. ${ }^{(13-17)}$ Since the transmitted power attenuates as the reciprocal of the distance, when the distances from the nodes to PUs are different, this mode leads to an imbalance in the harvested energy of nodes. Moreover, because the time slot is not fully utilized, the EH efficiency is low.

Motivated by the above analysis, in contrast to conventional underlay RF-CRNs, wherein SUs work in the harvesting-transmitting-inactive mode, in this paper, we propose a new energy and transmission mode for RF-CRNs with multiple SUs. In our mode, each SU harvests energy from the RF signals of each PU all the time except when transmitting data in the allocated time concurrently with the PU, namely, the "harvesting-transmission-harvesting" mode. In this way, the energy harvested by each SU can be maximized until the next data transmission. In addition, the total energy consumed by the SUs must be less than or equal to the total harvested energy, while the transmit power of SUs must be restricted to protect the PUs from interference.

Finally, quality of service (QoS) for multiple SUs is essential in many scenarios such as a wireless sensor network gathering data, monitoring events, and performing applications. To satisfy the QoS of SUs, we impose a minimum required throughput constraint on the transmitting process for each SU. In other words, our goal is to determine an optimal transmitting time and power allocation for SUs to maximize the achievable throughput of the RF-CRNs under interference, energy, and throughput constraints.

\subsection{Contributions}

The main contributions of this paper are summarized as follows:

- First, unlike the conventional harvesting-transmission-inactive mode, we design a new harvesting-transmission-harvesting mode that enables SUs to harvest sufficient energy for the subsequent data transmission.

- Second, we pay attention to guaranteeing the QoS of SUs in RF-CRNs. Given a prescribed minimum throughput threshold for each SU, we determine the optimal transmitting time and power allocation that maximize the expected throughput of RF-CRNs.

- Third, we achieve the optimal result by transforming the optimization problem into a convex optimization problem and then applying Lagrange multiplier methods.

- To verify the generality of the model, we investigate the impacts of typical parameters such as the transmitting power of the primary transmitter (PT), the maximum interference power of the primary receiver (PR), and the minimum throughput constraint for SUs on performance.

The rest of this paper is organized as follows. The system model for the EH-CRN is described in Sect. 2. The throughput analysis and throughput maximization solution are introduced in Sect. 3 and the simulated results are presented in Sect. 4. Finally, a conclusion is given in Sect. 5. 


\section{System Model}

We present a new model for RF-CRNs with multiple SUs as shown in Fig. 1. The secondary network consists of one access point (AP), $N$ secondary users depicted as $\mathrm{SU}_{k}(k=1,2, \ldots, N)$, which coexist with one PT and one PR. The AP has a fixed power supply, whereas each SU is powered by an energy harvester, which harvests green energy from RF signals of the PT. Assuming that the PT is always actively communicating with the PR, the SUs share the licensed spectrum with the PU under the interference constraint. There are EH links, data transmission links, and interference links in the model. The links between the PT and each SU are referred to as EH links with channel power coefficients $g_{E, k}(k=1,2, \ldots, N)$. The links between the AP and each $\mathrm{SU}_{k}(\mathrm{k}=1,2, \ldots, \mathrm{N})$ are data links with channel power gain coefficients $g_{D, k}(k=1,2, \ldots, N)$, and the links between the SUs and the PR are referred to as interference links with channel power gain coefficients $g_{I, k}(k=1,2, \ldots, N)$. All SUs are equipped with a single half-duplex omnidirectional antenna and a battery with finite capacity $E$ to store energy.

Meanwhile, as shown in Fig. 2, each frame with duration $T$ is allocated to $\mathrm{SU}_{k}(k=1,2, \ldots, N)$ for harvesting and transmission by TDMA. Unlike the conventional harvesting-transmissioninactive mode, we design a new harvesting-transmission-harvesting mode that can make $\mathrm{S} U_{\mathrm{k}}$ harvest energy after transmission rather than remain inactive. Specifically, $\mathrm{SU}_{k}$ continues to harvest energy from the RF signals of the PT all the time except during the transmission subslot. The transmission sub-slot allocated to $\mathrm{SU}_{k}$ is denoted as $\tau_{k}$ and the $\mathrm{EH}$ time for $\mathrm{SU}_{k}$ $(k=1,2, \ldots, N)$ is calculated as $\left(T-\tau_{k}\right)$. Hence, the total energy harvested by $\mathrm{SU}_{k}$ in frame $f$ is calculated as

$$
E_{H, k}^{(i)}=\eta_{k} g_{E, k} P_{T}\left(T-\tau_{k}\right), i=1,2, \ldots, f ; k=1,2, \ldots, N,
$$

where $\eta_{k}$ is the EH efficiency and $P_{T}$ is the transmission power of the PT. Note that the SUs share the spectrum with the PU in an underlay paradigm, which means that the SUs can perform concurrent transmission as long as the interference at the PR does not exceed a peak permissible threshold $P_{I}$ given as

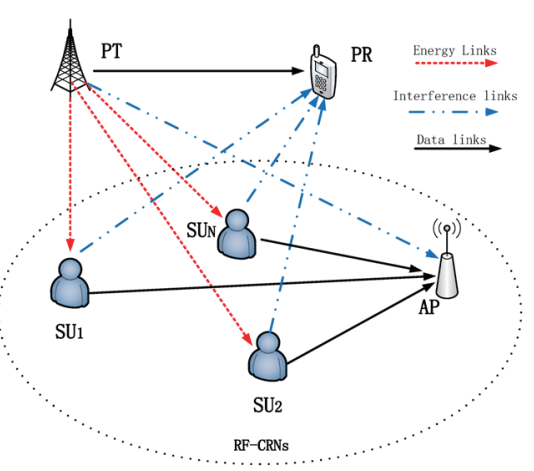

Fig. 1. (Color online) System model.

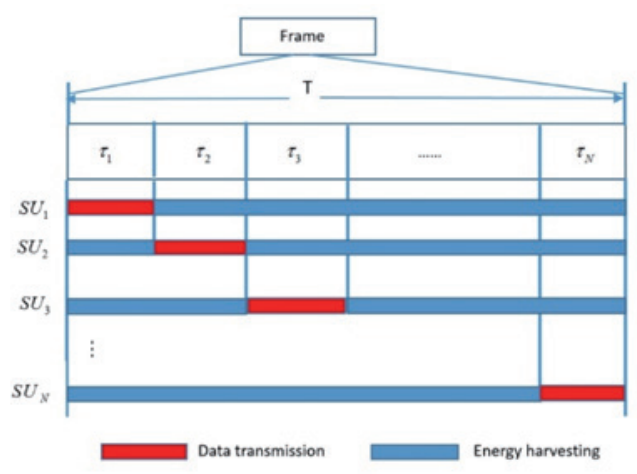

Fig. 2. (Color online) Frame structure. 


$$
P_{k} g_{I, k} \leq P_{I}, k=1,2, \ldots, N
$$

Since there is no sensing in an underlay paradigm, the energy required to receive and process information is negligible compared with the energy required for information transmission for convenience, and thus the SUs consume energy only for data transmission. Hence, the total energy consumption of an SU in frame $f$ is given by

$$
E_{C, k}^{(i)}=P_{k} \tau_{k}, i=1,2, \ldots, f ; k=1,2, \ldots, N
$$

The residual energy at the end of the previous frame $f-1$ can also be accumulated at the beginning of frame $f$ as the initial energy. Hence, by considering the harvested energy and consumed energy, the residual energy at the end of frame $f$ can be updated as

$$
E_{R, k}^{(f)}=E_{R, k}^{(f-1)}+E_{H, k}^{(f)}-E_{C, k}^{(f)},
$$

where $E_{R, k}^{(f-1)}$ is the former residual energy in frame $f-1$. Furthermore, supposing that the initial energy stored in the battery before communicating is $E_{0}, E_{R, k}^{(f)}$ is deduced to be

$$
E_{R, k}^{(f)}=E_{0}+\sum_{i=1}^{f}\left(E_{H, k}^{(i)}-E_{C, k}^{(i)}\right)
$$

By substituting Eqs. (1) and (3) into Eq. (5), we obtain

$$
E_{R, k}^{(f)}=E_{0}+\sum_{i=1}^{f}\left(\eta_{k} g_{E, k} P_{T}\left(T-\tau_{k}\right)-P_{k} \tau_{k}\right) .
$$

To guarantee the continuous communication of each $\mathrm{SU}, E_{R, k}^{(f)} \geq 0$ must be satisfied. Supposing that the maximum capacity of the battery is $E$ and surplus energy is discarded if $E_{R, k}^{(f)}>E$, then $E_{R, k}^{(f)} \leq E$ and we have

$$
0 \leq E_{0}+\sum_{i=1}^{f}\left(\eta_{k} g_{E, k} P_{T}\left(T-\tau_{k}\right)-P_{k} \tau_{k}\right) \leq E .
$$

\section{Throughput Analysis and Solution Method}

\subsection{Optimal problem formulation}

Given transmission power $P_{k}$ and the allocated time $\tau_{k}$, the achievable throughput of $\mathrm{SU}_{k}$ for the transmission from $\mathrm{SU}_{k}$ to the $\mathrm{AP}$ in one frame is 


$$
R_{k}\left(\tau_{k}, P_{k}\right)=\tau_{k} \log _{2}\left(1+\frac{P_{k} g_{D, k}}{P_{T} g_{0}+\sigma^{2}}\right), k=1,2, \ldots, N
$$

where $\frac{P_{k} g_{D, k}}{P_{T} g_{0}+\sigma^{2}}$ is the signal-to-noise ratio of the AP. We obtain the average achievable throughput of the RF-CRN as follows:

$$
R(\tau, \boldsymbol{P})=\frac{1}{T} \sum_{k=1}^{N} R\left(\tau_{k}, P_{k}\right)
$$

where $\boldsymbol{\tau}=\left[\tau_{1}, \tau_{2}, \ldots, \tau_{N}\right]$ and $\boldsymbol{P}=\left[P_{1}, P_{2}, \ldots, P_{N}\right]$ are the vectors of time allocation and power allocation, respectively. We set a minimum required rate $R_{\text {out }}>0$ for $\mathrm{SU}_{k}$, namely, the throughput constraint, $R_{k}\left(\tau_{k}, P_{k}\right) \geq R_{\text {out }}, \forall k$, to satisfy the QoS of SUs. Finally, we formulate the following maximum average throughput optimization problem.

$$
\begin{aligned}
& \max _{1 \leq k \leq N} R(\tau, P)=\frac{1}{T} \sum_{k=1}^{N} R_{k}\left(\tau_{k}, P_{k}\right) \\
& \text { s.t. } \mathrm{C} 1: P_{k} g_{I, k} \leq P_{I}, k=1,2, \ldots, N \\
& \mathrm{C} 2:-\frac{E_{0}}{f} \leq \eta_{k} g_{E, k} P_{T}\left(T-\tau_{k}\right)-P_{k} \tau_{k} \leq \frac{E-E_{0}}{f}, k=1,2, \ldots, N \\
& \mathrm{C} 3: \sum_{k=1}^{N} \tau_{k} \leq T \\
& \mathrm{C} 4: 0<\tau_{k}<T, 0<P_{k}<P_{\max }, k=1,2, \ldots, N \\
& \mathrm{C} 5: R_{k}\left(\tau_{k}, P_{k}\right) \geq R_{\text {out }}, \forall k
\end{aligned}
$$

$\mathrm{C} 1$ indicates that the transmission power of $\mathrm{SU}_{k}$ cannot exceed the tolerable inference power threshold $P_{I}$ at the PR, through which the PR is protected from interference, namely, the collision constraint. C2 indicates that for the allocated time $\tau_{k}$ and transmission power $P_{k}$, the energy consumed for data transmission cannot exceed the energy in the battery, namely, the energy causality constraint. $\mathrm{C} 3$ indicates that the total time consumed by $\mathrm{SU}_{k}$ must be less than or equal to the frame duration $T$. $\mathrm{C} 4$ is the consumed time constraint for each $\mathrm{SU}_{k}$ and $\mathrm{C} 4$ is the throughput constraint.

Obviously, Eq. (10) is a non-convex problem because of the product of the optimal variable $P_{k}$ and $\tau_{k}$ in C2. From Eq. (3), we can derive

$$
P_{k}=\frac{E_{C, k}^{(i)}}{\tau_{k}} .
$$


For convenience, we replace $E_{C, k}^{(i)}$ with $e_{k}$, and then we transform Eq. (8) into the following form:

$$
R_{k}\left(\tau_{k}, e_{k}\right)=\tau_{k} \log _{2}\left(1+\frac{e_{k}}{\tau_{k}} \gamma_{k}\right), k=1,2, \ldots, N
$$

where $\gamma_{k}=\frac{g_{D, k}}{P_{T} g_{0}+\sigma^{2}}$ is defined for convenience. Finally, we transform the optimization problem in Eq. (10) into the following problem with respect to $\boldsymbol{\tau}$ and $\boldsymbol{e}$, where $\boldsymbol{e}=\left[e_{1}, e_{2}, \ldots, e_{N}\right]$ is the energy consumed for $\mathrm{SU}_{k}$.

$$
\begin{aligned}
& \max _{1 \leq k \leq N} R(\tau, \boldsymbol{e})=\frac{1}{T} \sum_{k=1}^{N} R\left(\tau_{k}, e_{k}\right) \\
& \text { s.t. } \mathrm{Cl}^{\prime}: e_{k} g_{I, k} \leq \tau_{k} P_{I}, k=1,2, \ldots, N \\
& \mathrm{C} 2 \mathrm{a}^{\prime}:-\frac{E_{0}}{f} \leq \eta_{k} g_{E, k} P_{T}\left(T-\tau_{k}\right)-e_{k}, k=1,2, \ldots, N \\
& \mathrm{C} 2 \mathrm{~b}^{\prime}: \eta_{k} g_{E, k} P_{T}\left(T-\tau_{k}\right)-e_{k} \leq \frac{E-E_{0}}{f}, k=1,2, \ldots, N \\
& \mathrm{C} 3^{\prime}: \sum_{k=1}^{N} \tau_{k} \leq T \\
& \mathrm{C} 4^{\prime}: 0<\tau_{k}<T, k=1,2, \ldots, N \\
& \mathrm{C} 5^{\prime}: R\left(\tau_{k}, e_{k}\right) \geq R_{\text {out }}, \forall k
\end{aligned}
$$

Here, $\mathrm{C}^{\prime}$ and $\mathrm{C}^{\prime}$ are equivalent to $\mathrm{C} 3$ and $\mathrm{C} 4$ in Eq. (10), respectively, and $\mathrm{C} 2 \mathrm{a}^{\prime}$ and $\mathrm{C} 2 \mathrm{~b}^{\prime}$ are equivalent to $\mathrm{C} 2$.

Theorem 1: The achievable throughput $\mathrm{R}(\boldsymbol{\tau}, \boldsymbol{e})$ of the RF-CRN in Eq. (13) is a joint concave function of $\boldsymbol{\tau}$ and $\boldsymbol{e}$.

Proof: Please refer to Appendix A.

By applying Theorem 1, the objective function $R(\boldsymbol{\tau}, \boldsymbol{P})$ in Eq. (10) is converted into a concave function of $R(\boldsymbol{\tau}, \boldsymbol{e})$. After introducing $\boldsymbol{e}$, the energy causality constraint $\mathrm{C} 2$ is converted into affine functions as $\mathrm{C} 2 \mathrm{a}^{\prime}$ and $\mathrm{C} 2 \mathrm{~b}^{\prime}$. Thus, Eq. (13) is a convex optimization problem that can be solved by convex optimization techniques.

\subsection{Optimal resource allocation algorithm}

Equation (13) is a convex optimization problem and satisfies Slater's condition, so we can solve its dual problem instead. We first introduce the partial Lagrangian function of Eq. (13) with respect to $\mathrm{C} 2 \mathrm{a}^{\prime}, \mathrm{C} 2 \mathrm{~b}^{\prime}, \mathrm{C} 3^{\prime}$, and $\mathrm{C} 5^{\prime}$ : 


$$
\begin{aligned}
L(\tau, e, \lambda, u, v, w)= & \left(\sum_{k=1}^{N} \frac{1}{T}+\sum_{k=1}^{N} w_{k}\right) R_{k}\left(\tau_{k}, e_{k}\right) \\
& -\sum_{k=1}^{N} \lambda_{k}\left(-\frac{E_{0}}{f}-\eta_{k} g_{E, k} P_{T}\left(T-\tau_{k}\right)+e_{k}\right) \\
& -\sum_{i=1}^{N} u_{k}\left(\eta_{k} g_{E, k} P_{T}\left(T-\tau_{k}\right)-e_{k}-\frac{E-E_{0}}{f}\right) \\
& -v\left(\sum_{k=1}^{N} \tau_{k}-T\right)-\sum_{k=1}^{N} w_{k} R_{\text {out }},
\end{aligned}
$$

where $\lambda=\left[\lambda_{1}, \lambda_{2}, \ldots, \lambda_{N}\right], \boldsymbol{\mu}=\left[\mu_{1}, \mu_{2}, \ldots, \mu_{N}\right], \boldsymbol{w}=\left[w_{1}, w_{2}, \ldots, w_{N}\right] ; \lambda_{N}, \mu_{N}$, and $w_{N}$ are the nonnegative dual variables associated with $\mathrm{C} 2 \mathrm{a}^{\prime}, \mathrm{C} 2 \mathrm{~b}^{\prime}$, and $\mathrm{C}^{\prime}$, respectively; and $v$ is the nonnegative dual variable associated with $\mathrm{C} 3$ '.

Then the Lagrangian dual function is given as

$$
G(\lambda, u, v, w)=\max _{\tau, e \in D} L(\tau, e, \lambda, u, v, w)
$$

where $D$ is the set of $\boldsymbol{\tau}, \boldsymbol{e}$ associated with C1'. Finally, the dual problem of Eq. (13) is given by

$$
D(\lambda, u, v, w)=\min _{\lambda, u, v, w \geq 0} G(\lambda, u, v, w) .
$$

Theorem 2: For given $\lambda \geq 0, u \geq 0$, and $v \geq 0$, the optimal time and energy allocations are given by

$$
\begin{gathered}
\tau_{k}^{*}=-\frac{e_{k}^{*} \gamma_{k} W\left(\psi_{k}\right)}{W\left(\psi_{k}\right)+1}, k=1,2, \ldots, N, \\
e_{k}^{*}=\min \left(\left(\frac{\tau_{k}^{*}\left(\ln 2\left(\lambda_{k}-u_{k}\right)-\gamma_{k}\left(\frac{1}{T}+w_{k}\right)\right)}{\ln 2 \gamma_{k}\left(\lambda_{k}-u_{k}\right)}\right)^{+}, \frac{\tau_{k}^{*} P_{I}}{g_{I, k}}\right),
\end{gathered}
$$

where $W(\cdot)$ denotes the Lambert $W$ function, $\psi_{k}=-\exp \left(-\frac{\ln 2\left(\left(\lambda_{k}-u_{k}\right) \eta_{k} g_{E, k} P_{T}+v\right)}{w_{k}+1 / T}-1\right)$, and $(x)^{+} \triangleq \max (0, x)$.

Proof: Please refer to Appendix B.

Next, we compute the dual variables $\lambda, u, v$, and $w$ that minimize $D(\lambda, u, v)$ by using the subgradient method. The sub-gradients of $D(\lambda, u, v)$ at $\lambda_{k}, u_{k}, v$, and $w_{k}$ are calculated as

$$
\frac{\partial D}{\partial \lambda_{k}}=\frac{E_{0}}{f}+\eta_{k} g_{E, k} P_{T}\left(T-\tau_{k}\right)-e_{k},
$$




$$
\begin{gathered}
\frac{\partial D}{\partial u_{k}}=-\eta_{k} g_{E, k} P_{T}\left(T-\tau_{k}\right)+e_{k}+\frac{E-E_{0}}{f}, \\
\frac{\partial D}{\partial v}=-\left(\sum_{k=1}^{N} \tau_{k}-T\right), \\
\frac{\partial D}{\partial w_{k}}=R_{k}\left(\tau_{k}, e_{k}\right)-R_{\text {out }} .
\end{gathered}
$$

In the $n$th iteration, we update $\lambda_{k}, u_{k}, v$, and $w_{k}$ as follows:

$$
\begin{aligned}
& \lambda_{k}^{(n)}=\max \left\{0, \lambda_{k}^{(n-1)}+\alpha^{(n-1)}\left(\frac{\partial D}{\partial \lambda_{k}}\right)^{(n-1)}\right\}, \\
& u_{k}^{(n)}=\max \left\{0, u_{k}^{(n-1)}+\alpha^{(n-1)}\left(\frac{\partial D}{u_{k}}\right)^{(n-1)}\right\}, \\
& v=\max \left\{0, v^{(n-1)}+\alpha^{(n-1)}\left(\frac{\partial D}{v}\right)^{(n-1)}\right\}, \\
& w_{k}^{(n)}=\max \left\{0, w_{k}^{(n-1)}+\alpha^{(n-1)}\left(\frac{\partial D}{w_{k}}\right)^{(n-1)}\right\},
\end{aligned}
$$

where $\alpha^{(n-1)}$ denotes the step size in the $n$th iteration. The solution method is summarized as Algorithm 1.

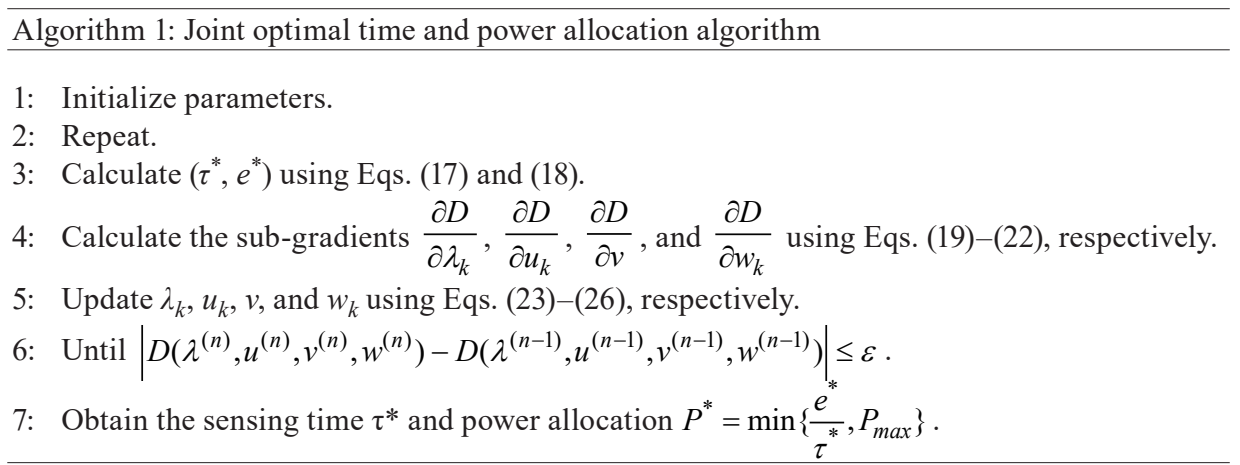




\section{Simulation Results}

Numerical results are shown to validate the performance of the proposed framework. The SUs are randomly placed in a circle with a radius of $10 \mathrm{~m}$ with the AP located at the center. The RF-CRNs coexist with a primary network that is deployed over an area with a radius of $100 \mathrm{~m}$. The harvested energy and the interference in the RF-CRNs depend on the location distribution of the SUs, so we assume that the channel power gain coefficients $g_{D, k}, g_{E, k}$, and $g_{I, k}$ are given by $g_{x, k}=1 /\left|d_{x, k}\right|^{\alpha}$, where $x=\{D, E, I\} . d_{x, k}$ and $\alpha$ are the distance and the path loss exponent, respectively.

The simulation parameters are set as $N=5, \alpha=2, f=1000, T=100 \mathrm{~ms}, \sigma^{2}=-140 \mathrm{dBm}$, $\eta_{k}=0.5, E_{0}=0.5 \mathrm{~J}, E=1 \mathrm{~J}$, and $P_{\max }=7 \mathrm{~W}$. Our model is similar to the one in Ref. 14 but with three important differences. First, the SUs work in the harvesting-transmitting mode through CDMA, as described in Ref. 14, whereas all SUs working in the harvesting-transmittingharvesting mode with TDMA can maximize the harvested energy. Second, the SUs save the energy in an infinite energy storage device, as shown in Ref. 14, whereas here, the SUs have a finite-capacity battery for storing energy, which is more realistic for practical application scenarios. Third, the RF-CRNs perform best-effort communication, and described in Ref. 14, whereas we make a minimum required throughput constraint on the transmitting process for each SU to ensure that the QoS of SUs is satisfied.

Figure 3 shows the throughput of the RF-CRNs under different values of $P_{T}, P_{I}$ given a prescribed minimum throughput threshold for each SU. The throughput clearly increases with increasing $P_{I}$, which is because increasing $P_{I}$ enlarges the feasible domain of $P_{k}$, further increasing $R(\boldsymbol{\tau}, \boldsymbol{P})$. However, $R(\boldsymbol{\tau}, \boldsymbol{P})$ decreases with increasing $P_{T}$. A larger $P_{T}$ means more harvested energy for SUs but also more interference with the AP. Thus, when the interference is large enough or $P_{I}$ is too small, the SUs cannot satisfy the prescribed minimum throughput threshold, the throughput dramatically decreases to near zero, and the system is interrupted.

Figures 4(a) and 4(b) show the energy status with different $P_{I}$. As shown in Fig. 3, $P_{k}$ increases with increasing $P_{I}$; thus, more energy is required for data transmission. Since the SUs

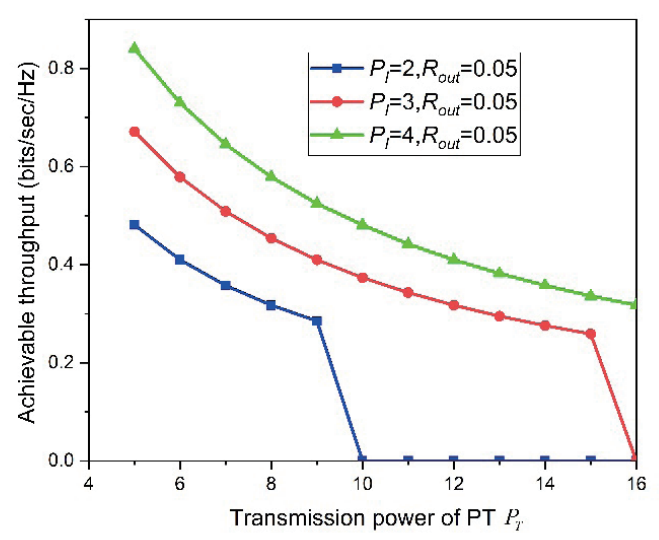

Fig. 3. (Color online) Throughput versus $P_{T}$. 
continuously harvest energy except during transmission, the harvested energy is abundant and the battery is full before each transmission of data.

Figure 5 shows the relation between the throughput $R(\boldsymbol{\tau}, \boldsymbol{P})$ and $P_{I}$. As shown in Figs. 3 and 4 , because $P_{k}$ increases with $P_{I}, R(\boldsymbol{\tau}, \boldsymbol{P})$ and the consumed energy increase accordingly. However, when $P_{I}$ is sufficiently large, $R(\boldsymbol{\tau}, \boldsymbol{P})$ cannot be further enhanced. In fact, $P_{k}$ cannot be infinite due to the finite battery capacity and the maximum power transmission constraint $P_{\max }$. Figure 6 depicts the maximum throughput for $R_{\text {out }}$ in different scenarios. It can be seen that $R(\boldsymbol{\tau}, \boldsymbol{P})$ and $R_{\text {out }}$ increase with $P_{I}$ at the same $P_{T}$. This is consistent with the fact that with increasing $P_{I}$, $R_{k}\left(\tau_{k}, P_{k}\right)$ and $R(\boldsymbol{\tau}, \boldsymbol{P})$ also increase as shown in Fig. 3. However, $R_{k}\left(\tau_{k}, P_{k}\right)$ finally tends to be stable due to the finite battery capacity and the existence of $P_{\max }$. Once the maximum limit $R_{\text {out }}$ is exceeded, the throughput dramatically decreases to near zero and the system is interrupted due to the minimum throughput constraints.

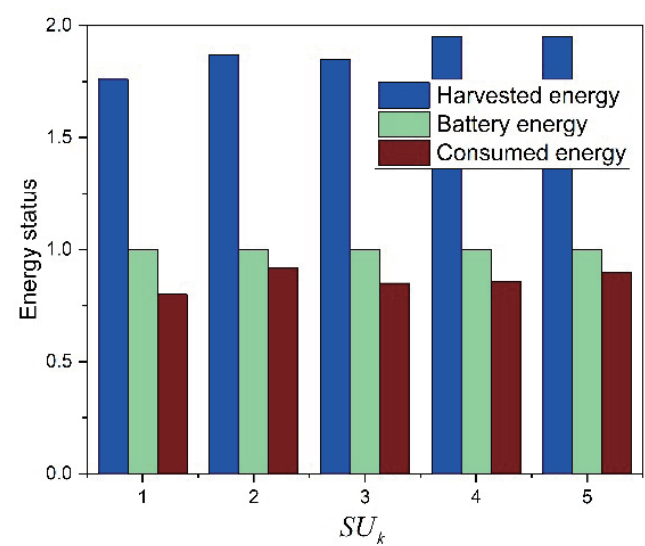

(a)

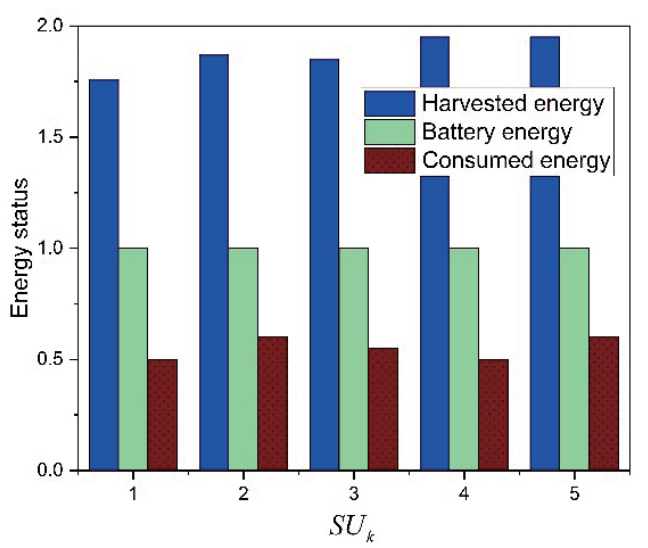

(b)

Fig. 4. (Color online) Energy status for SUs. (a) $P_{T}=12 \mathrm{~W}, P_{I}=4 \mathrm{~W}$. (b) $P_{T}=12 \mathrm{~W}, P_{I}=2 \mathrm{~W}$.

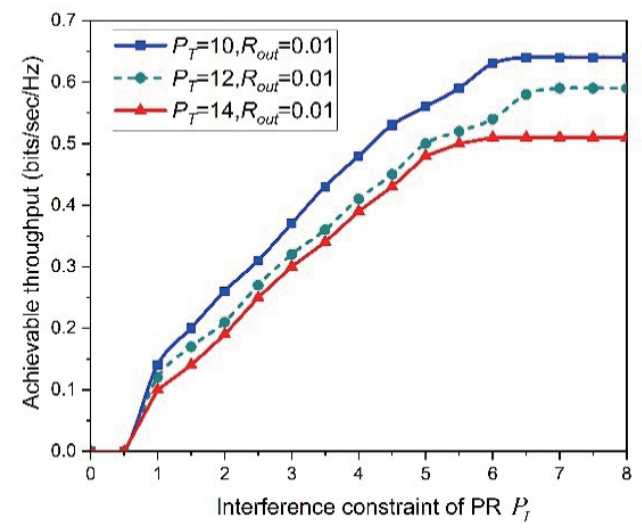

Fig. 5. (Color online) Throughput versus $P_{I}$.

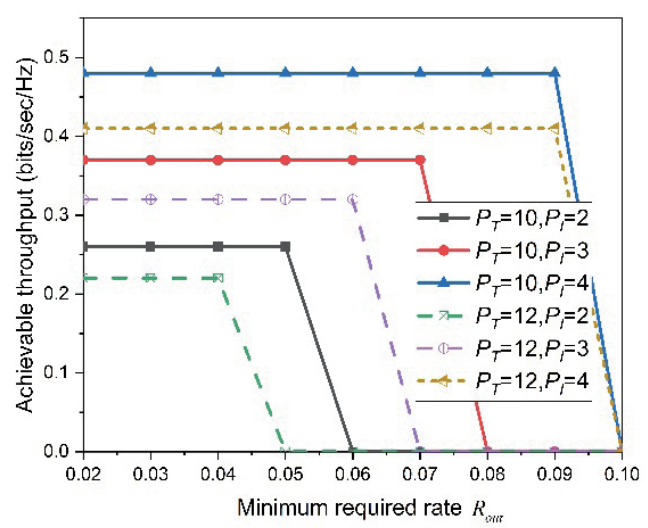

Fig. 6. (Color online) Throughput versus $R_{\text {out }}$. 


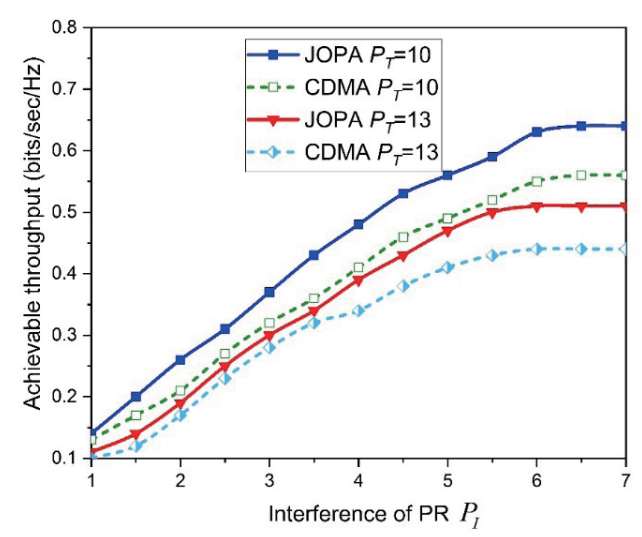

Fig. 7. (Color online) Throughput versus $P_{I}$ for different algorithms.

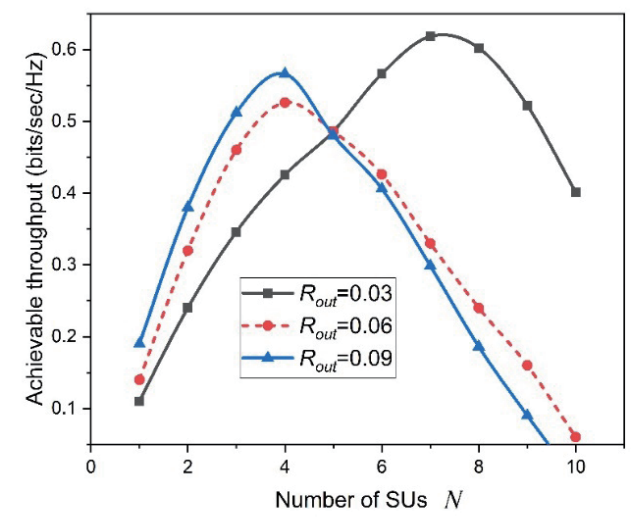

Fig. 8. (Color online) Throughput versus number of SUs $N$.

We compare our algorithm, namely, Algorithm 1, the joint optimal time and power allocation algorithm (JOPA), with the CDMA method in Ref. 14 for different levels of interference of the PR as shown in Fig. 7. It is obvious that JOPA achieves a larger throughput than CDMA as $P_{I}$ increases. This is mainly due to the harvested energies of the SUs being more sufficient in JOPA than in CDMA under the same $P_{T}$. With increasing $P_{I}$, the SUs can enhance their transmission powers adaptively as the harvested energies are sufficient, in contrast to in CDMA.

Figure 8 shows the throughput versus the number of SUs $N$ under different $R_{\text {out }}$. Indeed, when $N$ increases, the throughput will first increase and then gradually decrease until the system interrupts. This is because the achievable throughput $R(\boldsymbol{\tau}, \boldsymbol{P})$ of the RF-CRN is the sum of the throughputs $R_{k}\left(\tau_{k}, P_{k}\right)$ of $\mathrm{SU}_{k}(k=1,2, \ldots, N)$, as shown in Eqs. (8) and (9). Therefore, the greater the $N$, the greater the throughput of the RF-CRN. However, when $N$ continues to increase, the transmission time for each $\mathrm{SU}$ is reduced. Thereby, the achievable throughput $R_{k}\left(\tau_{k}, P_{k}\right)$ of $\mathrm{SU}_{k}$ $(k=1,2, \ldots, N)$ decreases and the QoS cannot be guaranteed, with the system interrupting.

\section{Conclusion}

In this paper, we presented a new harvesting-transmission-harvesting model for RF-CRNs. All SUs harvest energy from the RF signals of PUs all the time except when transmitting data in the allocated time. In this way, the energy harvested by each SU is always sufficient and can be maximized until the next data transmission, especially when the value of the interference constraint is large. Through the throughput constraint, we ensured transmission QoS for all SUs, which is essential in many applications, especially in wireless sensor networks performing data gathering, event monitoring, and other industrial applications. We achieved the optimal result by transforming the optimization problem into a convex optimization problem and then applying Lagrange multiplier methods. Through the maximization of harvesting energy and the minimum throughput constraint, our model can be expanded easily and used in many important applications such as large-scale CRNs, cognitive relay networks, and multi-hop CRNs. 


\section{Availability of Data and Material}

We declare that the materials described in the manuscript, including all relevant raw data, will be freely available to any scientist wishing to use them for non-commercial purposes, without breaching participant confidentiality.

\section{Competing Interests}

The authors declare that they have no competing interests.

\section{Funding}

This work was supported in part by the National Natural Science Foundation of China under Grant No. 61771410, and in part by the Scientific Research Fund of Sichuan Provincial Education Department under Grant No. 16ZB0185.

\section{Authors' contributions}

He Xiao conceived the presented idea, developed the theory, and performed the computations. Hong Jiang verified the analytical methods and analysis and supervised Li-Ping Deng in implementing the findings of this work. All authors discussed the results and contributed to the final manuscript, and all authors read and approved the final manuscript.

\section{Acknowledgments}

He Xiao would like to thank his supervisor who encouraged and supported him in this work. He Xiao, Hong Jiang, and Li-ping Deng are also very grateful to the reviewers and the journal employees.

\section{References}

1 S. Park and D. Hong: IEEE Trans. Wireless Commun.13 (2014) 1010. https://doi.org/10.1109/ TWC.2013.121713.130820

2 S. Yin, Z. Qu, and S. Li: IEEE J. Sel. Areas Commun. 33 (2015) 407. https://doi.org/10.1109/JSAC.2015.2391712

3 Y. Liu, S. A. Mousavifar, Y. Deng, C. Leung, and M. Elkashlan: IEEE Trans. Wireless Commun. 15 (2016) 2498.

4 D. T. Hoang, D. Niyato, P. Wang, and I. K. Dong: IEEE J. Sel. Areas Commun. 32 (2014) 2039. https://doi. org/10.1109/JSAC.2014.141108

5 X. Lu, P. Wang, D. Niyato, I. K. Dong, and Z. Han: IEEE Commun. Surveys Tutorials 17 (2015) 757. https://doi. org/10.1109/COMST.2014.2368999

6 C. Xu, M. Zheng, W. Liang, H. Yu, and Y. C. Liang: IEEE Commun. Lett. 20 (2016) 1148. https://doi. org/10.1109/LCOMM.2016.2547985

7 S. Yin, E. Zhang, L. Yin, and S. Li: Proc. IEEE Global Communications Conf. 2014 (GLOBECOM 2014) 1032-1037.

8 W. Chung, S. Park, S. Lim, and D. Hong: IEEE Trans. Wireless Commun. 13 (2014) 2601. https://doi. org/10.1109/TWC.2014.032514.130637

9 D. T. Hoang, D. Niyato, P. Wang, and D. I. Kim: IEEE J. Sel. Areas Commun.32 (2014) 2039. https://doi. org/10.1109/JSAC.2014.141108 
10 J. Jeya Pradha, S. Kalamkar, and A. Banerjee: IEEE Comm. Lett. 18 (2014) 1171. https://doi.org/10.1109/ LCOMM.2014.2323240

11 A. Kaushik, S. K. Sharma, S. Chatzinotas, B. Ottersten, and F. K. Jondral: IEEE Trans. Wireless Commun. 15 (2016) 3690. https://doi.org/10.1109/TWC.2016.2525986

12 S. Lee and R. Zhang: IEEE Trans. Cogn. Commun. Netw. 1 (2015) 335. https://doi.org/10.1109/ TCCN.2015.2508028

13 M. Zheng, C. Xu, W. Liang, and H. Yu: Electron. Lett. 52 (2016) 881. https://doi.org/10.1049/el.2015.4418

14 M. Zheng, L. Wei, and H. Yu: IEEE Syst. J. 12 (2016) 2395. https://doi.org/10.1109/JSYST.2016.2636278

15 D. Xu and Q. Li: IEEE Wireless Commun. Lett. 6 (2017) 294.https://doi.org/10.1109/LWC.2017.2676102

16 Y. Liu, S. A. Mousavifar, Y. Deng, C. Leung, and M. Elkashlan: IEEE Trans. Wireless Commun. 15 (2016) 2498. https://doi.org/10.1109/LWC.2017.2676102

17 C. Xu, M. Zheng, W. Liang, H. Yu, and Y. C. Liang: IEEE Trans. Wireless Commun. 16 (2017) 3561. https://doi. org/10.1109/TWC.2017.2684125

18 S. Yin, E. Zhang, Z. Qu, L. Yin, and S. Li: IEEE Trans. Wireless Commun. 13 (2014) 4693. https://doi. org/10.1109/TWC.2014.2322972

19 G. Zheng, Z. Ho, E. Jorswieck, and B. Ottersten: IEEE Trans. Signal Proc. 62 (2014) 2290. https://doi. org/10.1109/TSP.2014.2310433

20 Z. Wang, Z. Chen, B. Xia, L. Luo, and J. Zhou: IEEE Trans. Wireless Commun. 15 (2016) 2562. https://doi. org/10.1109/TWC.2015.2504581

21 S. S. Kalamkar, J. P. Jeyaraj, A. Banerjee, and K. Rajawat: IEEE Trans. Commun. 64 (2016) 3246. https://doi. org/10.1109/TCOMM.2016.2581162

22 S. Yin, Z. Qu, Z. Wang, and L. Li: IEEE Commun. Lett. 21 (2017) 128. https://doi.org/10.1109/ LCOMM.2016.2613537

23 K. Janghel and S. Prakriya: Proc. 2016 IEEE 17th Int. Workshop on Signal Processing Advances in Wireless Communications (SPAWC) 1-5.

24 Y. Liu, S. A. Mousavifar, Y. Deng, C. Leung, and M. Elkashlan: IEEE Trans. Wireless Commun. 15 (2016) 2498. https://doi.org/10.1109/TWC.2015.2504520

25 S. Singh, S. Modem, and S. Prakriya: IEEE Comm. Lett. 21 (2017) 1381. https://doi.org/10.1109/ LCOMM.2017.2666152

26 M. Mao, N. Cao, Y. Chen, and Y. Zhou: IEEE Wireless Commun. Lett. 4 (2015) 565. https://doi.org/10.1109/ LWC.2015.2462346

27 E. Chen, M. Xia, D. B. da Costa, and S. Aissa: IEEE Comm. Lett. 21 (2017) 1109. https://doi.org/10.1109/ LCOMM.2017.2655039

28 Z. Ding and H. V. Poor: IEEE Signal Process. Lett. 20 (2013) 1211. https://doi.org/10.1109/LSP.2013.2284800

29 Y. Gu and S. Aïssa: Proc. 2014 IEEE Int. Conf. Communications (ICC) 5378-5382.

30 Z. Yang, Z. Ding, P. Fan, and G. K. Karagiannidis: IEEE Trans. Veh. Technol. 65 (2016) 3828. https://doi. org/10.1109/TVT.2015.2443875

31 S. Boyd and L. Vandenberghe: Convex Optimization (Cambridge University Press, Cambridge, 2009) 7th ed., Chap. 2.

32 R. M. Corless, G. H. Gonnet, D. E. G. Hare, D. J. Jeffrey, and D. E. Knuth: Adv. Comput. Math. 5 (1996) 329. https://doi.org/10.1007/BF02124750

\section{Appendix A}

\section{Proof of Theorem 1}

Proof: In Eq. (13), $R_{k}\left(\tau_{k}, e_{k}\right)$ is the perspective transformation of the function $f\left(e_{k}\right) \triangleq \log _{2}\left(1+e_{k} \gamma_{k}\right)$. It is clear that $f\left(e_{k}\right)$ is a concave function of $e_{k}$ because the logarithmic function is concave. Because the perspective transformation preserves convexity, $R_{k}\left(\tau_{k}, e_{k}\right)$ is a concave function with respect to $\tau_{k}$ and $e_{k}$. Finally, because $R(\tau, \boldsymbol{e})$ is the sum of $N$ concave functions, it is also a concave function. ${ }^{(31)}$ 


\section{Appendix B}

\section{Proof of Theorem 2}

Proof: Because Eq. (13) is a convex optimization problem, there is strong duality between the primary and dual problems under Slater's condition. According to the Karush-Kuhn-Tucker (KKT) condition, the optimal solution must satisfy

$$
\begin{gathered}
\left.\frac{\partial L^{\prime}(\tau, e, \lambda, u, v, w)}{\partial \tau_{k}}\right|_{\tau_{k}=\tau_{k}^{*}}=0, k=1,2, \ldots, N, \\
\left.\frac{\partial L^{\prime}(\tau, e, \lambda, u, v, w)}{\partial e_{k}}\right|_{e_{k}=e_{k}^{*}}=0, k=1,2, \ldots, N .
\end{gathered}
$$

First, the partial derivative of $L(\tau, e, \lambda, u, v, w)$ with respect to $\tau_{k}$ can be calculated as follows:

$$
\begin{aligned}
& \frac{\partial L(\tau, e, \lambda, u, v, w)}{\partial \tau_{k}} \\
= & \frac{\partial\left\{\begin{array}{c}
\left(\sum_{k=1}^{N} \frac{1}{T}+\sum_{k=1}^{N} w_{k}\right) R_{k}\left(\tau_{k}, e_{k}\right)-\sum_{k=1}^{N} \lambda_{k}\left(-\frac{E_{0}}{f}-\eta_{k} g_{E, k} P_{T}\left(T-\tau_{k}\right)+e_{k}\right) \\
-\sum_{i=1}^{N} u_{k}\left(\eta_{k} g_{E, k} P_{T}\left(T-\tau_{k}\right)-e_{k}-\frac{E-E_{0}}{f}\right)-v\left(\sum_{k=1}^{N} \tau_{k}-T\right)-\sum_{k=1}^{N} w_{k} R_{\text {out }}
\end{array}\right\}}{\partial \tau_{k}} \\
= & \frac{\partial\left\{\begin{array}{c}
\left.\left(\sum_{k=1}^{N} \frac{1}{T}+\sum_{k=1}^{N} w_{k}\right) R_{k}\left(\tau_{k}, e_{k}\right)-\sum_{k=1}^{N} \lambda_{k}\left(\eta_{k} g_{E, k} P_{T} \tau_{k}\right)\right) \\
-\sum_{i=1}^{N} u_{k}\left(\eta_{k} g_{E, k} P_{T}\left(T-\tau_{k}\right)\right)-v\left(\sum_{k=1}^{N} \tau_{k}\right)
\end{array}\right\}}{\partial \tau_{k}} \\
= & \frac{\partial\left\{\begin{array}{c}
\left(\sum_{k=1}^{N} \frac{1}{T}+\sum_{k=1}^{N} w_{k}\right) R_{k}\left(\tau_{k}, e_{k}\right)-\sum_{k=1}^{N} \lambda_{k}\left(\eta_{k} g_{E, k} P_{T} \tau_{k}\right) \\
+\sum_{i=1}^{N} u_{k}\left(\eta_{k} g_{E, k} P_{T} \tau_{k}\right)-v\left(\sum_{k=1}^{N} \tau_{k}\right) \\
\partial\left\{\tau_{k}\right. \\
\left.\left(\sum_{k=1}^{N} \frac{1}{T}+\sum_{k=1}^{N} w_{k}\right) R_{k}\left(\tau_{k}, e_{k}\right)\right\}
\end{array}\right\}}{\partial \tau_{k}}-\lambda_{k} \eta_{k} g_{E, k} P_{T}+u_{k} \eta_{k} g_{E, k} P_{T}-v .
\end{aligned}
$$

In addition, we have 


$$
\frac{\partial R_{k}\left(\tau_{k}, e_{k}\right)}{\partial \tau_{k}}=\frac{\partial\left\{\tau_{k} \log _{2}\left(1+\frac{e_{k}}{\tau_{k}} \gamma_{k}\right)\right\}}{\partial \tau_{k}}=\frac{\ln \left(1+\frac{e_{k}}{\tau_{k}} \gamma_{k}\right)-\frac{e_{k} \gamma_{k}}{\tau_{k}+e_{k} \gamma_{k}}}{\ln 2}
$$

Then, we substitute Eqs. (29) and (30) into Eq. (27) to obtain

$$
\ln \left(1+\frac{e_{k} \gamma_{k}}{\tau_{k}}\right)-\frac{\frac{e_{k} \gamma_{k}}{\tau_{k}}}{1+\frac{e_{k} \gamma_{k}}{\tau_{k}}}=\frac{\ln 2\left(\left(\lambda_{k}-u_{k}\right) \eta_{k} g_{E, k} P_{T}+v\right)}{w_{k}+\frac{1}{T}}
$$

Subsequently, we make use of the Lambert $W$ function, which is the inverse of $f(x)=x \exp (x)$, to solve $\tau_{k}^{*}{ }^{(32)}$ Let $\beta=\frac{e_{k} \gamma_{k}}{\tau_{k}}$ and $\Upsilon=\frac{\ln 2\left(\left(\lambda_{k}-u_{k}\right) \eta_{k} g_{E, k} P_{T}+v\right)}{w_{k}+\frac{1}{T}}$. Then Eq. (29) can be converted into

$$
\begin{aligned}
& \ln (1+\beta)-\frac{\beta}{1+\beta}=\Upsilon \\
& \Rightarrow \ln (1+\beta)+\frac{1}{1+\beta}=\Upsilon+1 \\
& \Rightarrow(1+\beta)[\ln (1+\beta)-(\Upsilon+1)]+1=0 \\
& \Rightarrow(1+\beta) \ln \frac{(1+\beta)}{e^{(\Upsilon+1)}}+1=0 \\
& \Rightarrow \frac{(1+\beta)}{e^{(\Upsilon+1)}} \ln \frac{(1+\beta)}{e^{(\Upsilon+1)}}+\frac{1}{e^{(\Upsilon+1)}}=0 .
\end{aligned}
$$

Let $\zeta=\frac{(1+\beta)}{e^{(\Upsilon+1)}} \ln \frac{(1+\beta)}{e^{(\Upsilon+1)}}$; thus, $\frac{(1+\beta)}{e^{(\Upsilon+1)}}=e^{W(\zeta)}$. Then we obtain $\zeta=-e^{-\Upsilon-1}$ and $\beta=e^{W\left(-e^{-\Upsilon-1}\right)} e^{\Upsilon+1}-1$. Therefore, we have

$$
\begin{aligned}
& \tau_{k}=\frac{e_{k} \gamma_{k}}{\beta}=\frac{e_{k} \gamma_{k}}{e^{\Upsilon+1} e^{W\left(-e^{-\Upsilon-1}\right)}-1} \\
& \Rightarrow \tau_{k}=\frac{e_{k} \gamma_{k}}{e^{W\left(-e^{-\Upsilon-1}\right)}\left[e^{\Upsilon+1}-e^{-W\left(-e^{-\Upsilon-1}\right)}\right]} \\
& \Rightarrow \tau_{k}=\frac{e_{k} \gamma_{k} e^{-W\left(-e^{-\Upsilon-1}\right)}}{e^{\Upsilon+1}-e^{-W\left(-e^{-\Upsilon-1}\right)}} \\
& \Rightarrow \tau_{k}=-\frac{e_{k} \gamma_{k} W\left(-e^{-\Upsilon-1}\right)}{1+W\left(-e^{-\Upsilon-1}\right)} .
\end{aligned}
$$


Let $\psi_{k} \triangleq-e^{-\Upsilon-1}$. Then $\tau_{k}=-\frac{e_{k} \eta_{k} W\left(\psi_{k}\right)}{1+W\left(\psi_{k}\right)}$ and the finally obtained solution is given by Eq. (17).

Similarly, the partial derivative of $L(\tau, e, \lambda, u, v, w)$ with respect to $e_{k}$ can be calculated as follows:

$$
\begin{aligned}
& \frac{\partial L(\tau, e, \lambda, u, v, w)}{\partial e_{k}} \\
= & \frac{\partial\left\{\begin{array}{c}
\left(\sum_{k=1}^{N} \frac{1}{T}+\sum_{k=1}^{N} w_{k}\right) R_{k}\left(\tau_{k}, e_{k}\right)-\sum_{k=1}^{N} \lambda_{k}\left(-\frac{E_{0}}{f}-\eta_{k} g_{E, k} P_{T}\left(T-\tau_{k}\right)+e_{k}\right) \\
\left.-\sum_{i=1}^{N} u_{k}\left(\eta_{k} g_{E, k} P_{T}\left(T-\tau_{k}\right)-e_{k}-\frac{E-E_{0}}{f}\right)-v\left(\sum_{k=1}^{N} \tau_{k}-T\right)-\sum_{k=1}^{N} w_{k} R_{\text {out }}\right\}
\end{array}\right.}{\partial e_{k}} \\
= & \frac{\partial\left\{\left(\sum_{k=1}^{N} \frac{1}{T}+\sum_{k=1}^{N} w_{k}\right) R_{k}\left(\tau_{k}, e_{k}\right)-\sum_{k=1}^{N} \lambda_{k} e_{k}+\sum_{i=1}^{N} u_{k} e_{k}\right\}}{\partial e_{k}} \\
= & \frac{\partial\left\{\left(\sum_{k=1}^{N} \frac{1}{T}+\sum_{k=1}^{N} w_{k}\right) R_{k}\left(\tau_{k}, e_{k}\right)\right\}}{\partial e_{k}}-\lambda_{k}+u_{k} .
\end{aligned}
$$

We also have

$$
\frac{\partial R_{k}\left(\tau_{k}, e_{k}\right)}{\partial e_{k}}=\frac{\partial\left\{\tau_{k} \log _{2}\left(1+\frac{e_{k}}{\tau_{k}} \gamma_{k}\right)\right\}}{\partial e_{k}}=\frac{\gamma_{k}}{\left(1+\frac{\gamma_{k} e_{k}}{\tau_{k}}\right) \ln 2} .
$$

Lastly, by substituting Eqs. (34) and (35) into Eq. (28), we obtain

$$
e_{k}=\frac{\tau_{k}\left(\ln 2\left(\lambda_{k}-u_{k}\right)-\gamma_{k}\left(\frac{1}{T}+w_{k}\right)\right)}{\ln 2 \gamma_{k}\left(\lambda_{k}-u_{k}\right)} .
$$

Because $e_{k}$ is also subject to the interference power constraint $\mathrm{Cl}^{\prime}$, we have the optimal $e_{k}$ given by Eq. (18), thus proving Theorem 2. 
